\title{
Graph Theory and Sports Scheduling
}

\section{Richard Hoshino and Ken-ichi Kawarabayashi}

\section{Introduction}

The effects of global warming have been well documented, especially in recent years. As a result, the majority of countries have made a commitment to reducing their greenhouse gas emissions, including many whose national governments have made ambitious and unrealistic promises. Meeting these targets will require a coordinated effort from policymakers, businesses, and large industries, and numerous creative solutions will need to be implemented to achieve the desired goal. One potential solution is based on discrete mathematics, where combinatorial and graph-theoretic techniques are applied to scheduling optimization, leading to economic and environmental benefits.

There are many practical roles for mathematically optimal schedules that reduce total travel distance, including supply-chain logistics and airplane flight assignments. In this paper we describe how to optimize the regular-season schedule for Nippon Professional Baseball (NPB), Japan's most popular professional sports league, with annual revenues topping one billion U.S. dollars.

Given the authors' background as graph theorists, this research was motivated by the innocent question of whether NPB scheduling could be

Richard Hoshino is a mathematics tutor at Quest University Canada. His email address is richard.hoshino@questu. ca.

Ken-ichi Kawarabayashi is a professor at the National Institute of Informatics, Japan. His email address is k_keni ti @ ni i.ac.jp.

DOI: http://dx.doi.org/10.1090/noti1010 converted into a much simpler shortest-path problem. As we describe in this paper, the answer to this question is affirmative. Consequently, we have succeeded in generating the distance-optimal NPB regular-season schedule which retains all of the league's constraints that ensure competitive balance while reducing the total travel distance by $24.3 \%$, or nearly 70,000 kilometers, as compared to the 2010 season schedule.

To solve the NPB scheduling problem, we have generalized and extended the Traveling Tournament Problem (TTP), a well-known topic in sports scheduling [10]. Our research has produced five papers, [4], [5], [6], [7], [8], describing the theoretical aspects of the problem, providing various heuristics for generating distance-optimal intraleague and inter-league schedules, and applying the results to optimize the NPB league schedule.

Shortly after introducing the Traveling Tournament Problem [2], Easton et al. formed a consulting company to develop schedules for professional sports leagues. Their company, the Sports Scheduling Group, has received the contract to produce the regular-season schedule for Major League Baseball in six of the past seven years. Having now completed all of our research on NPB scheduling, our hope is to obtain the contract to produce future NPB regular-season schedules. We are excited by the possibility of sharing our expertise and passion with Nippon Professional Baseball, working in partnership with the league to produce schedules that save money and reduce greenhouse gas emissions, thus making an important contribution to Japan, both economically and environmentally. 


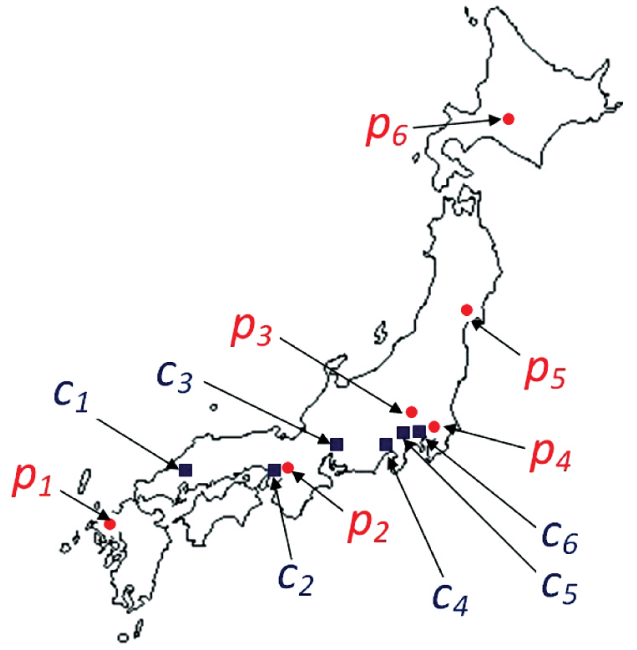

Figure 1. Location of the twelve teams in the NPB.

\section{Summary of Results}

Nippon Professional Baseball is split into the sixteam Pacific League and the six-team Central League. Each team plays 144 games during the regular season, with 120 intra-league games (against teams from their own league) and 24 inter-league games (against teams from the other league).

The location of each team's home stadium is given in Figure 1. For readability, we label each team as follows: the Pacific League teams are $p_{1}$ (Fukuoka), $p_{2}$ (Orix), $p_{3}$ (Saitama), $p_{4}$ (Chiba), $p_{5}$ (Tohoku), and $p_{6}$ (Hokkaido); and the Central League teams are $c_{1}$ (Hiroshima), $c_{2}$ (Hanshin), $c_{3}$ (Chunichi), $c_{4}$ (Yokohama), $c_{5}$ (Yomiuri), and $c_{6}$ (Yakult).

Specifically, each NPB team plays twelve home games and twelve away games against each of the other five teams in its league $(24 \times 5)$, in addition to two home games and two away games against all six teams in the other league $(4 \times 6)$. All twenty-four inter-league games take place during a common five-week stretch beginning in mid-May, right near the start of the season.

As in Major League Baseball, nearly all NPB games occur in sets of three games. Thus, we will adopt the same structure when building our distance-optimal schedule. For intra-league play, we have constructed a schedule where each team plays 40 sets of three games, with each team having an opponent in every time slot. (Similarly, for inter-league play, each team plays 12 sets of two games.)

For each team, we define a trip to be any pair of consecutive sets not occurring in the same venue, i.e., any situation where a team has to travel from one venue to another to play its next set of games. In Table 1 we list the total distance traveled by all teams under our mathematically optimal schedule, comparing that to the actual distance traveled by the teams during the 2010 season (not including games rescheduled due to weather). We also provide statistics for the number of total trips taken by the teams. Our optimal schedule reduces total distance by nearly 70,000 kilometers.

In this paper we present our analysis for the NPB intra-league problem. (We refer the reader to [8] for a detailed analysis of the inter-league problem.) In the following section we present the Multi-Round Balanced Traveling Tournament Problem, which generalizes the TTP and precisely models the scheduling parameters of the NPB. We then present our shortest-path reformulation and apply it to produce distance-optimal intra-league schedules for the Pacific and Central Leagues.

\begin{tabular}{c|rrc|ccc} 
& $\begin{array}{c}\text { Distance } \\
(2010)\end{array}$ & $\begin{array}{c}\text { Distance } \\
\text { (Optimal) }\end{array}$ & $\begin{array}{c}\text { Reduction } \\
\text { in Distance }\end{array}$ & $\begin{array}{c}\text { Trips } \\
(2010)\end{array}$ & $\begin{array}{c}\text { Trips } \\
\text { (Optimal) }\end{array}$ & $\begin{array}{c}\text { Reduction } \\
\text { in Trips }\end{array}$ \\
\hline Intra-League (PL) & 153,940 & 114,169 & $25.8 \%$ & 208 & 169 & $18.8 \%$ \\
Intra-League (CL) & 79,067 & 57,836 & $26.8 \%$ & 199 & 170 & $14.6 \%$ \\
Inter-League & 51,134 & 42,950 & $16.0 \%$ & 108 & 101 & $6.5 \%$ \\
\hline Total & 284,141 & 214,955 & $24.3 \%$ & 515 & 440 & $14.6 \%$
\end{tabular}

Table 1. The distance-optimal NPB schedule versus the actual 2010 regular-season schedule.

\section{The Multi-Round Balanced Traveling Tournament Problem}

Consider the intra-league schedule given in Table 2, which consists of $n=6$ teams each playing $k=4$ blocks of ten sets. Each block consists of two rounds, with each round having $n-1=5$ sets. Thus, each team plays a total of $k(2 n-2)=40$ sets. In this schedule, ${ }^{1}$ as with all subsequent schedules presented in this paper, home sets are marked in red.

Let $n$ and $k$ be positive integers. Let $D$ be the $n \times n$ distance matrix, where entry $D_{i, j}$ is the distance between the home stadiums of teams $i$ and $j$. By definition, $D_{i, j}=D_{j, i}$ for all $1 \leq i, j \leq n$, and all diagonal entries $D_{i, i}$ are zero.

For any pair $(n, k)$ and distance matrix $D$, the solution to the Multi-Round Balanced Traveling Tournament Problem (mb-TTP) is an intra-league tournament schedule that minimizes the total distance traveled by all $n$ teams, subject to the following conditions:

(a) The compactness condition: The tournament lasts $k(2 n-2)$ sets, i.e., $2 k$ rounds, where each team has one set scheduled in each time slot. (Thus $n$ must be even.)

(b) The each-round condition: Each pair of teams must play exactly once per round, with their matches in rounds $2 t-1$ and

\footnotetext{
${ }^{1}$ For example, team $p_{1}$ starts by playing a home set against $p_{2}$, followed by three consecutive road sets against $p_{5}, p_{3}$, $p_{4}$, then returning home to play three consecutive home sets against $p_{6}, p_{3}, p_{4}$, and so on.
} 


\begin{tabular}{|c|c|c|c|c|c|c|c|c|}
\hline Team & $\mathrm{R} 1$ & $\mathrm{R} 2$ & R3 & $\mathrm{R} 4$ & R5 & R6 & R7 & $\mathrm{R} 8$ \\
\hline$p_{1}$ & $\mathrm{p}_{2} p_{5} p_{3} p_{4} \mathrm{p}_{6}$ & $\mathbf{p}_{3} \mathbf{p}_{4} p_{6} p_{2} \mathbf{p}_{5}$ & $\mathrm{p}_{2} \mathrm{p}_{4} p_{6} p_{5} p_{3}$ & $\mathbf{p}_{6} \mathbf{p}_{5} \mathbf{p}_{3} p_{2} p_{4}$ & $p_{2} \mathrm{p}_{3} \mathrm{p}_{4} p_{5} p_{6}$ & $p_{4} \mathbf{p}_{5} \mathbf{p}_{6} \mathbf{p}_{2} p_{3}$ & $p_{6} p_{2} \mathbf{p}_{3} \mathbf{p}_{5} \mathbf{p}_{4}$ & $p_{3} p_{5} p_{4} \mathbf{p}_{6} \mathbf{p}_{2}$ \\
\hline$p_{2}$ & $p_{1} \mathbf{p}_{3} \mathbf{p}_{6} \mathbf{p}_{5} p_{4}$ & $p_{6} p_{5} \mathrm{p}_{4} \mathrm{p}_{1} p_{3}$ & $p_{1} \mathbf{p}_{3} \mathbf{p}_{4} p_{6} p_{5}$ & $p_{4} \mathrm{p}_{6} \mathrm{p}_{5} \mathrm{p}_{1} p_{3}$ & $\mathrm{p}_{1} \mathrm{p}_{4} p_{5} p_{6} p_{3}$ & $\mathrm{p}_{5} \mathrm{p}_{6} \mathrm{p}_{3} p_{1} p_{4}$ & $p_{3} \mathrm{p}_{1} \mathrm{p}_{5} \mathrm{p}_{4} p_{6}$ & $p_{5} p_{4} \mathrm{p}_{6} \mathrm{p}_{3} p_{1}$ \\
\hline$p_{3}$ & $p_{4} p_{2} \mathbf{p}_{1} \mathbf{p}_{6} \mathbf{p}_{5}$ & $p_{1} p_{6} p_{5} \mathbf{p}_{4} \mathbf{p}_{2}$ & $\mathrm{p}_{6} p_{2} p_{5} \mathrm{p}_{4} \mathrm{p}_{1}$ & $\mathrm{p}_{5} p_{4} p_{1} p_{1} \mathrm{p}_{2}$ & $\mathbf{p}_{5} p_{1} p_{6} p_{4} \mathbf{p}_{2}$ & $\mathbf{p}_{6} \mathbf{p}_{4} p_{2} p_{5} \mathbf{p}_{1}$ & $\mathbf{p}_{2} p_{4} p_{1} \mathbf{p}_{6} \mathbf{p}_{5}$ & $\mathrm{p}_{1} p_{6} p_{5} p_{2} \mathrm{p}_{4}$ \\
\hline$p_{4}$ & $\mathrm{p}_{3} p_{6} p_{5} \mathrm{p}_{1} \mathrm{p}_{2}$ & $\mathrm{p}_{5} p_{1} p_{2} p_{3} \mathrm{p}_{6}$ & $\mathrm{p}_{5} p_{1} p_{2} p_{3} \mathbf{p}_{6}$ & $\mathrm{p}_{2} \mathrm{p}_{3} p_{6} p_{5} \mathrm{p}_{1}$ & $\mathbf{p}_{6} p_{2} p_{1} \mathbf{p}_{3} \mathbf{p}_{5}$ & $\mathrm{p}_{1} p_{3} p_{5} p_{6} \mathbf{p}_{2}$ & $p_{5} \mathbf{p}_{3} \mathbf{p}_{6} p_{3} p_{1}$ & $p_{6} \mathrm{p}_{2} \mathrm{p}_{1} \mathrm{p}_{5} p_{3}$ \\
\hline$p_{5}$ & $p_{6} \mathbf{p}_{1} \mathbf{p}_{4} p_{2} p_{3}$ & $p_{4} \mathbf{p}_{2} \mathbf{p}_{3} \mathbf{p}_{6} p_{1}$ & $p_{4} p_{6} \mathrm{p}_{3} \mathrm{p}_{1} \mathrm{p}_{2}$ & $p_{3} p_{1} p_{2} \mathbf{p}_{4} \mathbf{p}_{6}$ & $p_{3} \mathrm{p}_{6} \mathrm{p}_{2} \mathrm{p}_{1} p_{4}$ & $p_{2} p_{1} \mathbf{p}_{4} \mathbf{p}_{3} p_{6}$ & $\mathbf{p}_{4} \mathbf{p}_{6} p_{2} p_{1} p_{3}$ & $\mathrm{p}_{2} \mathbf{p}_{1} p_{3} p_{4} p_{6}$ \\
\hline$p_{6}$ & $\mathbf{p}_{5} \mathbf{p}_{4} p_{2} p_{3} p_{1}$ & $\mathrm{p}_{2} \mathrm{p}_{3} \mathrm{p}_{1} p_{5} p_{4}$ & $p_{3} \mathrm{p}_{5} \mathrm{p}_{1} \mathrm{p}_{2} p_{4}$ & $p_{1} p_{2} \mathbf{p}_{4} \mathrm{p}_{3} p_{5}$ & $p_{4} p_{5} \mathrm{p}_{3} \mathrm{p}_{2} \mathrm{p}_{1}$ & $p_{3} p_{2} p_{1} \mathbf{p}_{4} \mathbf{p}_{5}$ & $\mathrm{p}_{1} p_{5} p_{4} p_{3} \mathrm{p}_{2}$ & $\mathbf{p}_{4} \mathbf{p}_{3} p_{2} p_{1} \mathbf{p}_{5}$ \\
\hline
\end{tabular}

Table 2. A 40-set (120-game) intra-league schedule for the NPB Pacific League.

$2 t$ taking place at different venues (for all $1 \leq t \leq k)$.

(c) The at-most-three condition: No team may have a home stand or road trip lasting more than three sets.

(d) The no-repeat condition: A team cannot play against the same opponent in two consecutive sets.

(e) The diff-two condition: Let $H_{i, s}$ and $R_{i, s}$ be the number of home and away sets played by team $i$ within the first $s$ sets. Then $\left|H_{i, s}-R_{i, s}\right| \leq 2$ for all $(i, s)$ with $1 \leq i \leq n$ and $1 \leq s \leq k(2 n-2)$.

For example, one can quickly verify that Table 2 satisfies all five conditions. When calculating the total distance, we will assume that each team begins the tournament at home and returns home after having played their last away set. Furthermore, when a team is scheduled for a road trip consisting of multiple away sets, the team doesn't return to their home city but rather proceeds directly to their next away venue. ${ }^{2}$ The mb-TTP is an extension of the well-known NP-hard Traveling Salesman Problem, asking for an optimal schedule linking venues that are close to one another.

We now present an algorithm for solving the mb-TTP, for any $k \geq 1$, by reformulating it as a shortest-path problem on a directed graph. We will create a source node and a sink node and link them to numerous vertices in a graph whose (weighted) edges represent the possible blocks that can appear in an optimal schedule. We then apply Dijkstra's Algorithm [1] to find the path of minimum weight between the source and the sink, which is an $O(|V| \log |V|+|E|)$ graph search algorithm that can be applied to any graph or digraph with nonnegative edge weights.

\section{Shortest-Path Reformulation}

By definition, a block is a two-round tournament schedule satisfying the conditions of the mb-TTP,

\footnotetext{
${ }^{2}$ Thus, after the first block of ten sets, team $p_{1}$ will have traveled a total distance of $D_{p_{1}, p_{5}}+D_{p_{5}, p_{3}}+D_{p_{3}, p_{4}}+D_{p_{4}, p_{1}}+$ $D_{p_{1}, p_{6}}+D_{p_{6}, p_{2}}+D_{p_{2}, p_{1}}$.
}

with each of the $n$ teams playing $2(n-1)$ sets of games. To solve the mb-TTP, we first compute the set of blocks that can appear in a distanceoptimal tournament. We then introduce a simple "concatenation matrix" to check whether two precomputed blocks can be joined together to form a multiblock schedule without violating any of the five conditions of the mb-TTP. As we will explain, to determine whether two (feasible) blocks $B_{1}$ and $B_{2}$ can be concatenated, it suffices to check just the last two columns of $B_{1}$ and the first two columns of $B_{2}$.

Each column of a block represents a set consisting of $\frac{n}{2}$ different matches, with each match specifying the two teams as well as the stadium/venue. Thus, a match identifies the home team and away team, not just each team's opponent. For any column, there are $\left(\begin{array}{c}n \\ n / 2\end{array}\right)$ ways to select the home teams. Also, there are $\left(\begin{array}{c}n \\ n / 2\end{array}\right) \cdot\left(\frac{n}{2}\right)$ ! ways to specify the matches of any column, since there are $\left(\frac{n}{2}\right)$ ! ways to map any choice of the $\frac{n}{2}$ home teams to the unselected $\frac{n}{2}$ away teams to decide the set of $\frac{n}{2}$ matches. Hence, there are $m=\left(\begin{array}{c}n \\ n / 2\end{array}\right)^{2} \cdot\left(\frac{n}{2}\right)$ ! different ways we can specify the matches of the first column and the home teams of the second column. For $n=6$, we have $m=\left(\begin{array}{l}6 \\ 3\end{array}\right)^{2} \times 3 !=2400$.

There are $m$ ways that the first two columns of a block can be chosen as described above, with the first column listing matches and the second column listing home teams. Now use any method, such as a lexicographic ordering, to index these $m$ options with the integers from 1 to $m$. By symmetry, there are $m$ different ways we can specify the last two columns of a block, with the last column listing matches and the second-to-last column listing home teams. Thus, we use the same scheme to index these $m$ options. To avoid confusion, we write the home teams column in binary form, with 1 representing a home game and 0 representing an away game. 

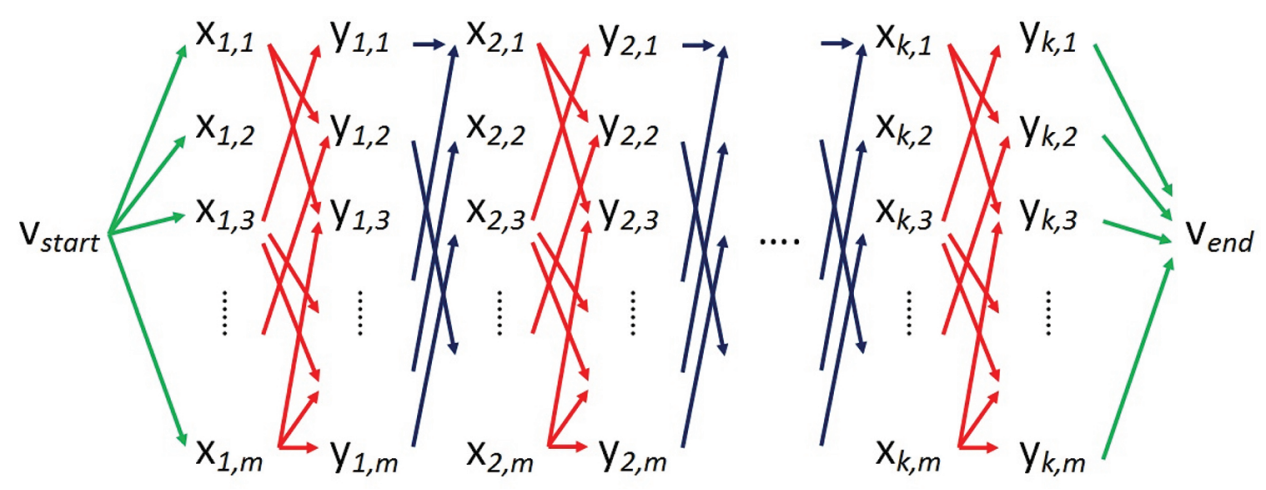

Figure 2. Reformulation of the $k$-block mb-TTP as a shortest-path problem.

For example, $\left(p_{5}, p_{3}, p_{2}, p_{6}, p_{1}, p_{4}\right)^{T}$ is one of the 120 possibilities for the matches column, and $(0, \mathbf{1}, \mathbf{1}, 0, \mathbf{1}, 0)^{T}$ is one of the 20 possibilities for the home teams column. We remark that if we listed the column of opponents rather than the column of matches, there would be only $\frac{120}{2^{3}}=15$ unique columns, corresponding to the 15 perfect matchings of the complete graph $K_{6}$.

For the NPB Pacific League, there exists some integer $q$ (with $1 \leq q \leq 2400$ ) that is the index of the instance where the home teams column is $(0,1,1,0,1,0)^{T}$ and the matches column is $\left(p_{5}\right.$, $\left.p_{3}, p_{2}, p_{6}, p_{1}, p_{4}\right)^{T}$. Similarly, there exists some $r$ (with $1 \leq r \leq 2400$ ) that is the index of the instance where the two columns are $\left(p_{2}, p_{1}, p_{6}, p_{5}\right.$, $\left.p_{4}, p_{3}\right)^{T}$ and $(\mathbf{1}, \mathbf{1}, 0,0,0, \mathbf{1})^{T}$. In Table 2 the last two columns of block 1 have index $q$ and the first two columns of block 2 have index $r$.

For each pair $\left(u_{1}, u_{2}\right)$, with $1 \leq u_{1}, u_{2} \leq m$, define $C_{u_{2}, u_{1}}$ to be the $n \times 4$ concatenation matrix where the first two columns list the home teams and matches with index $u_{2}$, and the next two columns list the matches and home teams with index $u_{1}$. For the indices $x$ and $y$ from the previous paragraph, we have

$$
C_{q, r}=\left[\begin{array}{llll}
0 & p_{5} & p_{2} & \mathbf{1} \\
\mathbf{1} & p_{3} & p_{1} & \mathbf{1} \\
\mathbf{1} & p_{2} & p_{6} & 0 \\
0 & p_{6} & p_{5} & 0 \\
\mathbf{1} & p_{1} & p_{4} & 0 \\
0 & p_{4} & p_{3} & \mathbf{1}
\end{array}\right]
$$

Note that $C_{q, r}$ has no row with four home sets, no row with four away sets, and no row with the same opponent appearing in columns 2 and 3. As we describe in Theorem 1 , these three properties are a necessary and sufficient condition for whether two feasible blocks can be concatenated to produce a multiblock schedule satisfying the conditions of the mb-TTP.

Before we proceed with Theorem 1, let us explain the role of $m$ and $C_{u_{2}, u_{1}}$ in the construction of our directed graph. Let $G$ consist of a source vertex $v_{\text {start }}$, a sink vertex $v_{\text {end }}$, and vertices $x_{t, u}$ and $y_{t, u}$ defined for each $1 \leq t \leq k$ and $1 \leq u \leq m$.

We now describe how these edges are connected with a pictorial representation of $G$ in Figure 2 . For notational simplicity, denote $v_{1} \rightarrow v_{2}$ as the directed edge from $v_{1}$ to $v_{2}$.

(i) For each $1 \leq u \leq m$, add the edge $v_{\text {start }} \rightarrow$ $x_{1, u}$.

(ii) For each $1 \leq u \leq m$, add the edge $y_{k, u} \rightarrow$ vend.

(iii) For each $1 \leq t \leq k$ and for each $1 \leq u_{1}, u_{2} \leq$ $m$, add the edge $x_{t, u_{1}} \rightarrow y_{t, u_{2}}$ iff there exists a (feasible) block for which the first two columns have index $u_{1}$ and the last two columns have index $u_{2}$.

(iv) For each $1 \leq t \leq k-1$ and for each $1 \leq u_{1}, u_{2} \leq m$, add the edge $y_{t, u_{2}} \rightarrow x_{t+1, u_{1}}$ iff the concatenation matrix $C_{u_{2}, u_{1}}$ has no row with four home sets, no row with four away sets, and no row with the same opponent appearing in columns 2 and 3.

The following theorem [4] shows that the $k$-block mb-TTP can be reformulated in a graph-theoretic context for any $k \geq 1$.

Theorem 1. Every feasible solution of the $m b$-TTP can be described by a path from $v_{\text {start }}$ to $v_{\text {end }}$ in graph $G$. Conversely, any path from $v_{\text {start }}$ to $v_{\text {end }}$ in $G$ corresponds to a feasible solution of the $m b-T T P$.

Having constructed our digraph, we now assign a weight to each edge using the distance matrix $D$ so that the shortest path (i.e., path of minimum total weight) from $v_{\text {start }}$ to $v_{\text {end }}$ corresponds to the optimal solution of the mb-TTP that minimizes the total distance traveled by the $n$ teams.

For any block, we define its in-distance to be the total distance traveled by the $n$ teams within that block, i.e., starting from set 1 and ending at set $2(n-1)$. Note that the in-distance does not include the distance traveled by the teams heading to the venue of set 1 or from the venue of set $2(n-1)$. We will use this definition in part (c) below: 


\begin{tabular}{|c|c|c|c|c|c|c|c|c|}
\hline Team & $\mathrm{R} 1$ & $\mathrm{R} 2$ & $\mathrm{R} 3$ & $\mathrm{R} 4$ & R5 & R6 & $\mathrm{R} 7$ & $\mathrm{R} 8$ \\
\hline$c_{1}$ & $\mathrm{c}_{2} \mathrm{C}_{5} \mathcal{C}_{4} \mathcal{C}_{6} \mathcal{C}_{3}$ & $\mathrm{C}_{4} \mathrm{C}_{6} \mathrm{C}_{3} \mathcal{C}_{2} \mathcal{C}_{5}$ & $c_{3} \mathrm{C}_{2} \mathrm{C}_{5} \mathrm{C}_{6} \mathcal{C}_{4}$ & $C_{5} c_{6} \mathrm{c}_{4} \mathrm{c}_{3} \mathcal{C}_{2}$ & $c_{4} c_{2} \mathrm{C}_{3} \mathrm{c}_{6} \mathrm{c}_{5}$ & $C_{3} C_{6} C_{5} C_{4} c_{2}$ & $c_{4} C_{2} C_{3} C_{6} C_{5}$ & $c_{3} C$ \\
\hline$c_{2}$ & $C_{1} \mathrm{C}_{3} \mathrm{C}_{5} \mathcal{C}_{4} \mathcal{C}_{6}$ & $C_{5} \mathrm{C}_{4} \mathrm{C}_{6} \mathrm{C}_{1} C_{3}$ & $c_{4} C_{1} \mathrm{c}_{3} \mathrm{C}_{5} \mathrm{c}_{6}$ & $C_{3} C_{5} C_{6} \mathrm{c}_{4} \mathrm{c}_{1}$ & $C_{3} \mathrm{c}_{1} \mathrm{c}_{6} \mathrm{C}_{5} C_{4}$ & $C_{6} C_{5} \mathrm{C}_{4} \mathrm{C}_{3} C_{1}$ & $C_{3} \mathrm{C}_{1} \mathrm{c}_{6} \mathrm{C}_{5} \mathcal{C}_{4}$ & $C_{6} C_{5} C_{4} C_{3} C_{1}$ \\
\hline$c_{3}$ & $\mathrm{c}_{4} C_{2} C_{6} C_{5} \mathbf{c}_{1}$ & $\mathbf{c}_{6} \mathbf{C}_{5} C_{1} C_{4} \mathbf{C}_{2}$ & $\mathrm{c}_{1} C_{6} C_{2} C_{4} \mathrm{c}_{5}$ & $\mathbf{C}_{2} \mathbf{c}_{4} c_{5} c_{1} c_{6}$ & $\mathbf{c}_{2} c_{5} c_{1} \mathbf{c}_{4} \mathbf{c}_{6}$ & $\mathrm{c}_{1} C_{4} C_{6} C_{2} \mathrm{c}_{5}$ & $\mathrm{C}_{2} C_{5} C_{1} \mathbf{C}_{4} \mathbf{C}_{6}$ & $\mathbf{C}_{1} C_{4} C_{6} C_{2} \mathbf{c}_{5}$ \\
\hline$c_{4}$ & $C_{3} \mathrm{C}_{6} \mathrm{C}_{1} \mathrm{c}_{2} C_{5}$ & $c_{1} C_{2} \mathrm{C}_{5} \mathrm{C}_{3} c_{6}$ & $\mathrm{c}_{2} C_{5} C_{6} \mathrm{C}_{3} \mathrm{C}_{1}$ & $\mathrm{c}_{6} \mathcal{C}_{3} C_{1} C_{2} \mathbf{c}_{5}$ & $\mathrm{c}_{1} C_{6} C_{5} C_{3} \mathbf{C}_{2}$ & $\mathrm{c}_{5} \mathrm{C}_{3} C_{2} C_{1} \mathrm{c}_{6}$ & $\mathrm{c}_{1} c_{6} c_{5} c_{3} \mathrm{c}_{2}$ & $\mathrm{C}_{5} \mathrm{C}$ \\
\hline$c_{5}$ & $\mathrm{c}_{6} \mathcal{C}_{1} \mathcal{C}_{2} \mathrm{c}_{3} \mathrm{c}_{4}$ & $\mathrm{c}_{2} C_{3} C_{4} C_{6} \mathrm{c}_{1}$ & $\mathrm{c}_{6} \mathbf{c}_{4} C_{1} C_{2} C_{3}$ & $\mathrm{c}_{1} \mathrm{c}_{2} \mathbf{c}_{3} c_{6} c_{4}$ & $C_{6} \mathbf{C}_{3} \mathbf{c}_{4} C_{2} C_{1}$ & $C_{4} \mathrm{C}_{2} \mathrm{C}_{1} \mathrm{C}_{6} \mathcal{C}_{3}$ & $C_{6} \mathbf{C}_{3} \mathbf{c}_{4} \mathcal{C}_{2} C_{1}$ & $\mathrm{C}_{6} \mathrm{C}_{3}$ \\
\hline$c_{6}$ & $c_{5} c_{4} C_{3} C_{1} C_{2}$ & $C_{3} C_{1} C_{2} C_{5} C_{4}$ & $C_{5} \mathrm{C}_{3} \mathrm{C}_{4} C_{1} C_{2}$ & $C_{4} \mathrm{C}_{1} \mathrm{C}_{2} \mathrm{C}_{5} C_{3}$ & $\mathrm{C}_{5} \mathrm{C}_{4} \mathcal{C}_{2} C_{1} C_{3}$ & $\mathrm{c}_{2} \mathrm{c}_{1} \mathrm{c}_{3} \mathcal{C}_{5} \mathcal{C}_{4}$ & $\mathrm{C}_{5} \mathrm{C}_{4} C_{2} C_{1} C_{3}$ & \\
\hline
\end{tabular}

Table 3. The distance-optimal intra-league schedule for the NPB Central League.

\begin{tabular}{c|ccc|ccc} 
& $\begin{array}{c}\text { Distance } \\
(2010)\end{array}$ & $\begin{array}{c}\text { Distance } \\
\text { (Optimal) }\end{array}$ & $\begin{array}{c}\text { Reduction } \\
\text { in Distance }\end{array}$ & $\begin{array}{c}\text { Trips } \\
(2010)\end{array}$ & $\begin{array}{c}\text { Trips } \\
\text { (Optimal) }\end{array}$ & $\begin{array}{c}\text { Reduction } \\
\text { in Trips }\end{array}$ \\
\hline$p_{1}$ & 33352 & 21143 & $36.6 \%$ & 35 & 27 & $22.9 \%$ \\
$p_{2}$ & 24128 & 18713 & $22.4 \%$ & 34 & 29 & $14.7 \%$ \\
$p_{3}$ & 20885 & 19498 & $6.6 \%$ & 34 & 28 & $17.6 \%$ \\
$p_{4}$ & 23266 & 16606 & $28.6 \%$ & 36 & 29 & $19.4 \%$ \\
$p_{5}$ & 23710 & 17975 & $24.2 \%$ & 37 & 29 & $21.6 \%$ \\
$p_{6}$ & 28599 & 20234 & $29.2 \%$ & 32 & 27 & $15.6 \%$ \\
\hline Total & 153940 & 114169 & $25.8 \%$ & 208 & 169 & $18.8 \%$
\end{tabular}

Table 4. Comparison of intra-league schedules for the Pacific League.

(a) For each $1 \leq u \leq m$, the weight of edge $v_{\text {start }} \rightarrow x_{1, u}$ is the total distance traveled by the $\frac{n}{2}$ teams making the trip from their home city to the venue of their opponent in set 1 .

(b) For each $1 \leq u \leq m$, the weight of edge $y_{k, u} \rightarrow v_{\text {end }}$ is the total distance traveled by the $\frac{n}{2}$ teams making the trip from the venue of their opponent in set $2 k(n-1)$ back to their home city.

(c) For each $1 \leq t \leq k$, and for each $1 \leq$ $u_{1}, u_{2} \leq m$, the weight of edge $x_{t, u_{1}} \rightarrow y_{t, u_{2}}$ is the minimum in-distance of a block, selected among all blocks for which the first two columns have index $u_{1}$ and the last two columns have index $u_{2}$.

(d) For each $1 \leq t \leq k-1$ and for each $1 \leq u_{1}, u_{2} \leq m$, the weight of edge $y_{t, u_{2}} \rightarrow$ $x_{t+1, u_{1}}$ is the total distance traveled by the teams that travel from their match in set $2 t(n-1)$ to their match in set $2 t(n-1)+1$, where the last two columns of the $t^{\text {th }}$ block have index $u_{2}$ and the first two columns of the $(t+1)^{\text {th }}$ block have index $u_{1}$.

To illustrate (d), consider the first two blocks in Table 2, where the last two columns of block 1 have index $q$ and the first two columns of block 2 have index $r$. When we concatenate these two blocks, the weight of edge $y_{1, q} \rightarrow x_{2, r}$ is the total distance traveled by the teams from their matches in set 10 to their matches in set 11 . This sum equals
$D_{p_{3}, p_{1}}+D_{p_{1}, p_{4}}+D_{p_{4}, p_{3}}$, the distances traveled by teams $p_{2}, p_{5}$, and $p_{6}$, respectively.

By this construction, we have produced a weighted digraph. In part (c), suppose there exist two blocks $B$ and $B^{\prime}$ for which the first two columns have index $u_{1}$ and the last two columns have index $u_{2}$. If the in-distance of $B$ is less than the in-distance of $B^{\prime}$, then block $B^{\prime}$ cannot be a block in an optimal solution, since we can just replace $B^{\prime}$ by $B$ to create a feasible solution with a lower objective value. This trivial observation, based on Bellman's Principle of Optimality, allows us to assign the minimum in-distance as the weight of edge $x_{t, u_{1}} \rightarrow y_{t, u_{2}}$, for all $1 \leq u_{1}, u_{2} \leq m$. As a result, we have a digraph $G$ on $2 m k+2$ vertices and at most $2 m+(2 k-1) m^{2}$ edges, with a unique weight for each edge. Combined with the previous theorem, we have established the following.

Theorem 2. Let $P=v_{\text {start }} \rightarrow x_{1, p_{1}} \rightarrow y_{1, q_{1}} \rightarrow$ $x_{2, p_{2}} \rightarrow y_{2, q_{2}} \rightarrow \cdots \rightarrow x_{k, p_{k}} \rightarrow y_{k, q_{k}} \rightarrow v_{\text {end }}$ be $a$ shortest path in $G$ from $v_{\text {start }}$ to $v_{\text {end }}$, i.e., a path that minimizes the total weight. For each $1 \leq t \leq k$, let $B_{t}$ be the block of minimum in-distance selected among all blocks for which the first two columns have index $p_{t}$ and the last two columns have index $q_{t}$. Then the multiblock schedule $S=B_{1}, B_{2}, \ldots, B_{k}$, created by concatenating the $k$ blocks consecutively, is an optimal solution of the $m b$-TTP.

\section{Application to the NPB}

Therefore, we have shown that the mb-TTP is isomorphic to finding the shortest weighted path in the directed graph $G$. For the case $n=6$, we can show that $G$ consists of $4800 k+2$ vertices and $2400+2400+2618520 k+1486320(k-1)=$ $4104840 k-1481520$ edges. Given the distance matrices for the NPB Pacific and Central Leagues, we can determine the appropriate weights for each edge in $G$ to determine the shortest path from $v_{\text {start }}$ to $v_{\text {end }}$ using Dijkstra's Algorithm, which in turn generates the distance-optimal schedule. We wrote our code using Maplesoft; for each league, Maplesoft produced the distance-optimal intraleague schedule after five hours of computation 
time. The large majority of the running time was spent determining the correct edge weight for part (c) of our construction. For more information, we refer the reader to [7].

The optimal Pacific League intra-league schedule appears in Table 2; the optimal Central League intra-league schedule appears in Table 3.

In Table 1 we produced an overall summary of the results. In Table 4 we provide a teamby-team breakdown for the NPB Pacific League, comparing our distance-optimal schedule to the actual schedule played by the teams during the 2010 regular season.

In addition to the significant $25.8 \%$ reduction in total distance traveled, we also remark that this is a more equitable schedule. In the 2010 NPB intraleague schedule, team $p_{1}$ traveled nearly 12, 500 kilometers more than team $p_{3}$. Under our schedule, the difference between the most traveled and least traveled would reduce to just 4,500 kilometers. For the Central League, the difference between the most traveled and least traveled would reduce from 7, 500 kilometers to just 4,000 kilometers.

\section{Implementation}

Naturally, there are additional factors involved with the actual scheduling of NPB games at these home stadiums. For example, one of the ballparks hosts a three-day concert each August, and another ballpark is used as the locale of the national high school baseball tournament. Hence those teams must play away games on those particular days. In many sports leagues, rival teams play "derby matches" that need to be scheduled on particular days to optimize revenue and that are often dictated by the wishes of television broadcasters to add drama and boost TV ratings. These constraints must be taken into account when producing an optimal schedule that can be implemented by NPB to ensure no conflicts occur and that the schedule is the best possible for all parties involved.

\section{Epilogue}

After the publication of our initial results, the Nippon Professional Baseball (NPB) League invited us (the authors) to visit their head office. Over the course of three meetings in September 2012, we consulted the NPB and helped them design the Central League's 2013 intra-league schedule [3].

When we met with the NPB head scheduler, we learned that the league has additional scheduling constraints, such as the "revenue-balancing" requirement that each team play the same number of weekend home games, weekday home games, weekend road games, and weekday road games. In [9], we describe how we fully solved this TTP-variant for the case $n=6$, and helped the NPB design the intra-league schedule for the 2013 Central
League, reducing the number of total trips by 12 and cutting over six thousand kilometers of total travel distance, as compared to the schedule from the previous season.

We look forward to partnering with the NPB once again, and hope to have the opportunity to help this league produce future regular-season schedules that will result in annual win-wins for the people of Japan, both economically and environmentally.

\section{References}

[1] E. W. DiJKstra (1959), A note on two problems in connexion with graphs, Numerische Mathematik, 1, 269-271.

[2] K. Easton, G. Nemhauser, and M. Trick (2001), The traveling tournament problem: description and benchmarks, Proceedings of the 7th International Conference on Principles and Practice of Constraint Programming, pp. 580-584.

[3] S. Hesse, Canadian scientist uses math to green Japanese baseball, http://www.japantimes.co. jp/life/2012/11/25/environment/canadianscientist-uses-math-to-green-japanesebaseba11/, Japan Times, 2012.

[4] R. Hoshino and K. KaWARABAyashi (2011), The multi-round balanced traveling tournament problem, Proceedings of the 21st International Conference on Automated Planning and Scheduling (ICAPS), pp. 106-113.

[5] (2011), The inter-league extension of the traveling tournament problem and its application to sports scheduling, Proceedings of the 25th AAAI Conference on Artificial Intelligence, pp. 977-984.

[6] __ (2011), The distance-optimal inter-league schedule for Japanese pro baseball, Proceedings of the ICAPS 2011 Workshop on Constraint Satisfaction Techniques for Planning and Scheduling Problems (COPLAS), 71-78.

[7] (2011), A multi-round generalization of the traveling tournament problem and its application to Japanese baseball, European Journal of Operational Research, 215, 481-497.

[8] _ (2011), Scheduling bipartite tournaments to minimize total travel distance, Journal of Artificial Intelligence Research, 42, 91-124.

[9] __ Balancing the Traveling Tournament Problem for Weekday and Weekend Games, Proceedings of the 2013 AAAI Conference, to appear.

[10] G. Kendall, S. KNust, C. C. Ribeiro, and S. URRUTIA (2010), Scheduling in sports: an annotated bibliography, Computers and Operations Research, 37, $1-19$. 